\title{
Two-Stage Robotic Crystal Mounting of Protein Crystals for X-Ray Data Collection
}

\author{
Atanas Georgiev and Peter K. Allen
}

\begin{abstract}
We present a new microrobotic system for a crystallographic task called protein crystal mounting, which is the transfer of a crystal from its growth solution onto a tool designed to hold it for $\mathrm{X}$-ray data collection. The system operates autonomously using feedback from a camera looking at the workspace through a microscope. Visual tracking is employed to monitor the location of the selected crystal and control a range of actuators for successful extraction. The task is performed in two stages: First, the crystal is taken from its drop into a pipette and is transported into a cryoprotecting liquid. Second, while in the cryoprotector, it is transferred from the pipette onto a tool to be used for data collection. Key features of this approach are the increased robustness of the system and the avoidance of the damaging effect of direct exposure of the crystal to room conditions. This work is part of a larger effort we have directed at the automation of the high-throughput crystallographic pipeline.
\end{abstract}

\section{INTRODUCTION}

The completion of the Human Genome Project in 2003 [1] was a major milestone in the history of the life sciences and is having a revolutionary impact in medicine where research is starting to look at genetic causes for a wide range of maladies on molecular level. The project, whose most widely-known outcome was the sequencing of the human DNA, was the first undertaking of such calibre in biology, involved a coordinated international participation and took 13 years to complete.

Following on its footsteps is the ongoing Structural Genomics Project, jump-started in the United States by the National Institutes of Health with the Protein Structure Initiative, currently in its second phase [2]. This initiative aims at determining and cataloguing the three-dimensional atomic-level structure of proteins, the products of genes. Experimentally determined structures can be used along with sequence data and bioinformatics methods to expand the structural coverage to the majority of proteins. The resulting database is expected to have an unprecedented beneficial impact on biological, biomedical and clinical research in multiple ways, including by highlighting the relationships of structure to function and disease, directing structurebased drug design [3] and refining our understanding of evolutionary relationships between species.

The structure determination of a single protein is however a long and tedious process. One of the principal methods for doing so is X-ray macromolecular crystallography (the other being nuclear magnetic resonance spectroscopy), which is

Atanas Georgiev and Peter K. Allen are with the Department of Computer Science, Columbia University, New York, NY 10027, USA $\{$ atanas, allen\}@cs. columbia.edu

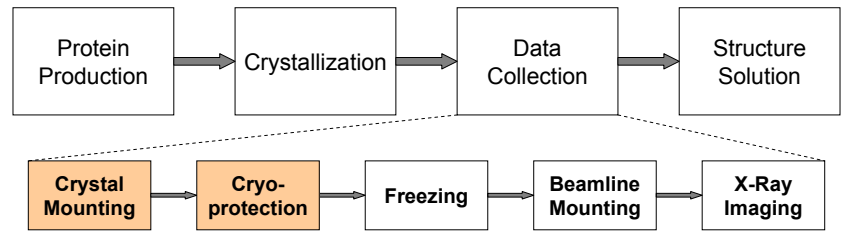

Fig. 1. A simplified crystallography pipeline. The top row shows a basic outline of the main steps in the pipeline. The bottom row is a detailed expansion of the data collection step.

roughly illustrated in Fig. 1. It starts with protein expression, where large quantities of protein are obtained by coaxing a bacteria to overproduce it. Next, the protein is crystallized by mixing it with a reagent (which differs among proteins) in a small drop and incubating inside chambers in crystallization plates (e.g. Linbro plates). When a crystal of large enough size and high enough quality is obtained, it is placed in a synchrotron for X-ray imaging and the images are processed by software methods to arrive at the spatial arrangement of the atoms. For data collection to occur, the crystal is transferred from its incubation chamber to a synchrotron, which involves extracting the crystal from its drop, immersing it into a cryoprotecting liquid, cryogenically freezing it and eventually placing it on the beam line of the synchrotron for imaging (Fig. 1, bottom row). During these steps, the crystal is placed on a special mounting tool that allows the appropriate handling.

As part of the Protein Structure Initiative, the research centers involved have streamlined the process into a highthroughput pipeline and have developed and utilized the necessary technology for its automation. By now, many of the steps have been automated, however, there still remain some that are performed manually. In this paper, we are addressing one such specific task, called crystal mounting, in conjunction with the following step, called cryoprotection (both highlighted in Fig. 1). Crystal mounting is currently performed by skilled crystallographers and is simply described as moving a selected crystal from its growth solution to a suitable mounting tool for data collection on a synchrotron. The mounting tool preferred by crystallographers is usually a cryogenic loop, which is a loop made of a thin $(10 \mu \mathrm{m}$ or $20 \mu \mathrm{m})$ thread of nylon glued to the tip of a metal pin. Manually mounting a crystal in a loop requires time, patience and excellent motor skills.

The automation of crystal mounting necessitates the development of strategies and tools for automated and fast 

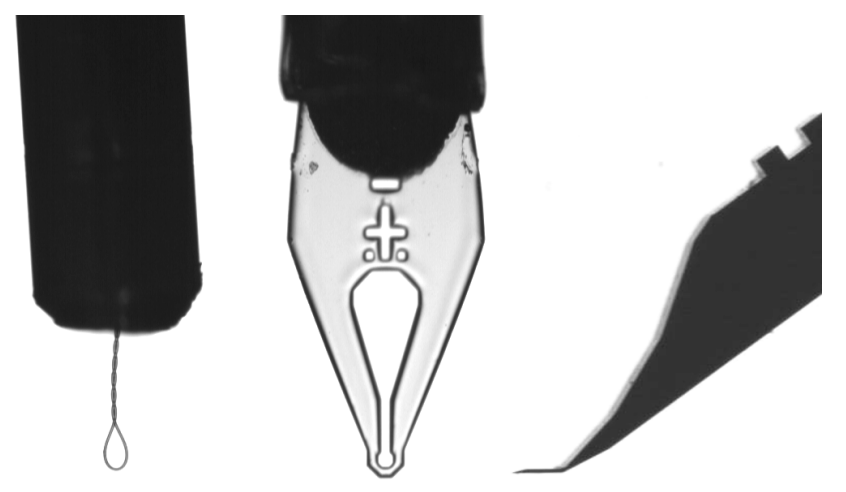

Fig. 2. Crystal mounting tools: a cryogenic loop (left), a micromount (center) and a microshovel (right).

manipulation of protein crystals. This is quite challenging because of the unique combination of factors involved. Protein crystals are small (sizes of interest are between $25 \mu \mathrm{m}$ and $1000 \mu \mathrm{m})$ and quite fragile. They are very sensitive to environmental variations, especially temperature. Their growth environment is a $0.1-5 \mu$ d droplet of liquid which dehydrates in a few minutes once exposed to room conditions.

Our work is aimed at augmenting precise instrumentation with computer vision techniques to effect accurate and robust crystal manipulation without the need for extensive analysis of the physics of grasping or a detailed knowledge of the environment. In a previous work [4], we proposed an integrated control system operating under a high-resolution optical microscope for crystal mounting based on a novel customdesigned tool we call a microshovel. In this work, we present a two-stage solution which combines the crystal mounting and cryoprotection steps and is designed to increase the robustness of the pickup as well as better preserving the crystal.

\section{RELATED WORK}

Throughout the history of protein crystallography, numerous ideas were suggested for how to mount a crystal and contain it during X-ray data collection. Glass capillaries have been used for growing protein crystals and data collection since early in the development of the field [5], [6]. The cryogenic loop (Fig. 2, left) eventually became the dominant tool used for mounting because of a number of attractive properties, such as reduced background scattering and flexible material which helps avoid damage to the crystals [7].

More recently, new tools, such as the micromount (Fig. 2, center) and the microshovel (Fig. 2, right), have been developed which improve on the loop in various ways. For example, some of the attractive features of the micromount, introduced by Thorne et al [8], are that its design facilitates the automation of the beamline mounting and alignment steps, it is made of material that reduces the amount of background X-ray scattering during data collection and its fabrication technology allows for precise control of the size of the sample hole. In our earlier work on crystal mounting [4], we presented the microshovel and described its use in an automated crystal mounting system. The tool is made out of silicon by using a micro-electro-mechanical systems (MEMS) technology and features reduced background scattering of the X-rays, rigidity for improved machine control and numerous precise shapes and sizes. For detailed description of the microshovel and its fabrication, we refer the reader to [9].

Automation of the related steps in the X-ray crystallography pipeline has been pursued, though the most popular targets seem to have been sample preparation [10] and beamline mounting and alignment [11], [12]. Telerobotic techniques have also been investigated [13].

In terms of the mechanics of the actual manipulation, in the rapidly progressing field of microrobotics quite a few diverse ideas have been pursued. Various kinds of microgrippers have been proposed [14]. Optical trapping by a laser has been successfully used for both direct and indirect cell manipulation [15]. A micromanipulation tool based on adhesive forces has been demonstrated [16]. Methods exploiting magnetic and electrostatic forces are also being used.

Unfortunately, not many of these approaches can be easily applied to protein crystallography. Microgrippers pose the risk of structural damage to the fragile crystals. Optical trapping has the potential to damage the crystals because of excessive heat. Adhesive forces can not be relied upon because of the drastic variations of the composition of the protein crystals and their environments. Dielectrophoresis actuates all objects within the electromagnetic field making it difficult to isolate an individual target.

One of the major advances in robotics over the last 20 years is the visual control of robotic manipulators [17]. The advent of fast and inexpensive digital imaging technology has allowed camera systems to be integrated as part of a closedloop feedback control system [18]. Visual servoing strategies have been successfully implemented at the microscale level for manipulation of known micro-electromechanical systems with calibrated devices [19], [20]. Visual servoing has also been successfully used for biological cell injection [21].

Visual servoing is classified into two main approaches [22], [17], [23]. The first one [24], [25], based on the computation of a 3-D Cartesian error, requires a perfect model of the object and a calibrated camera to obtain unbiased pose estimation. In the second approach, the pose estimation is omitted and the control loop is directly closed in the image space. That ensures local convergence and stability in presence of modeling errors and noise perturbations [26]. In this work, we use the image-based approach, even though our system is partially calibrated.

\section{HARDWARE SyStem AND SETUP}

The robotic crystal mounting system we have built is shown in Fig. 3. It consists of a personal computer and the following hardware devices: a microscope, a video camera, a motorized stage, a micromanipulator, a microinjector. The video camera is connected to the computer via a Hauppauge 


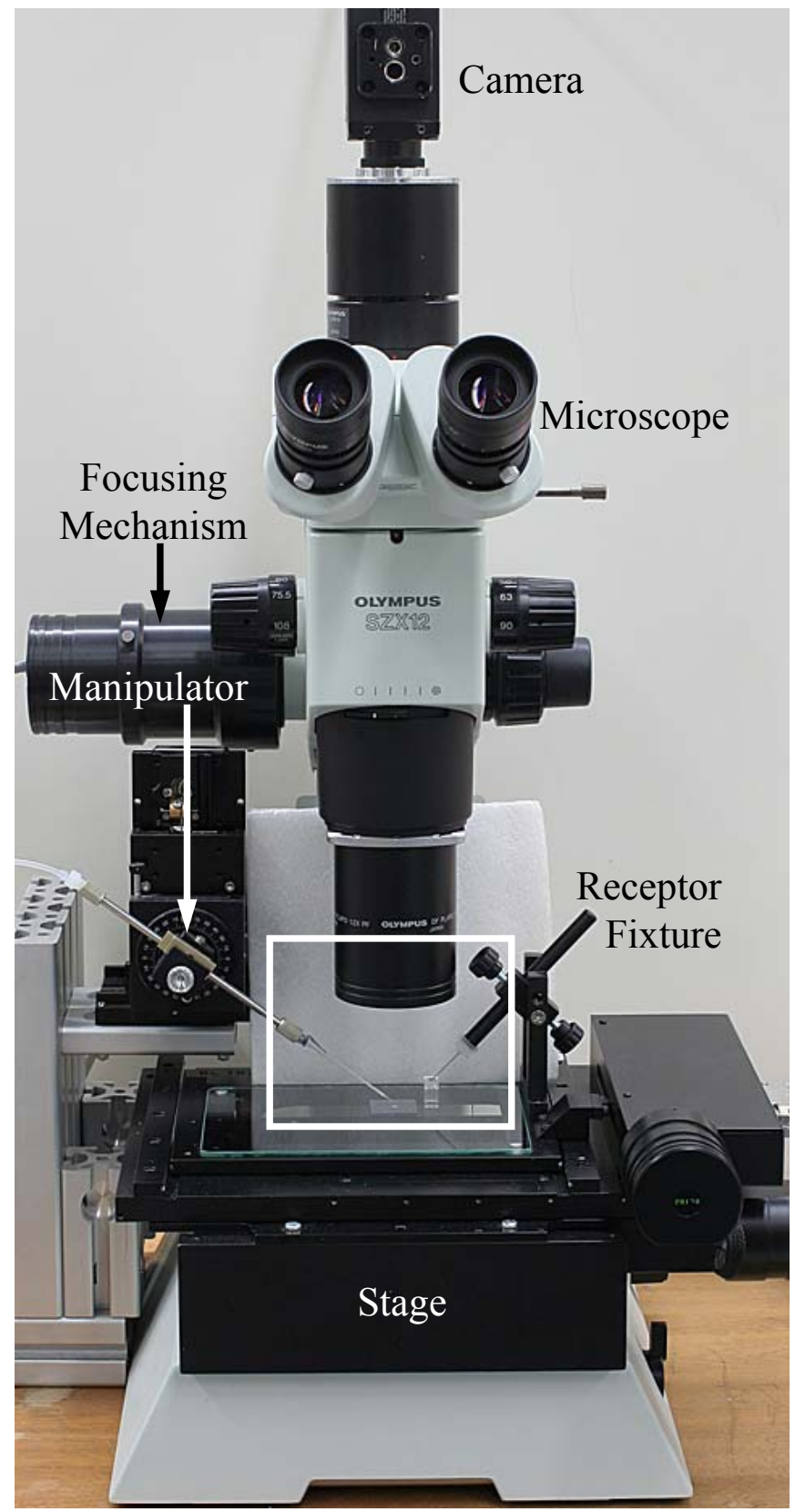

Fig. 3. Robotic workstation for crystal mounting. A closeup of the rectangular region is shown in Fig. 4.

WinTV frame grabber and each actuator is connected to the computer via an RS-232 serial interface.

The microscope, which serves as the base of our platform, is an Olympus SZX-12. It is an optical transmitted-light microscope, provides magnification from $8.4 \mathrm{x}$ to $108 \mathrm{x}$ and has a computer-controlled focusing mechanism. The workspace is observed by a Sony XC-77 video camera which is mounted on the microscope and provides continuous video feed to the computer. The computer, running the software component of the system, analyzes the scene and controls the actuators.

The motorized stage is a Prior Proscan. It provides two degrees of freedom (DOF) of horizontal motion and is used to position the relevant part of the workspace in the field of

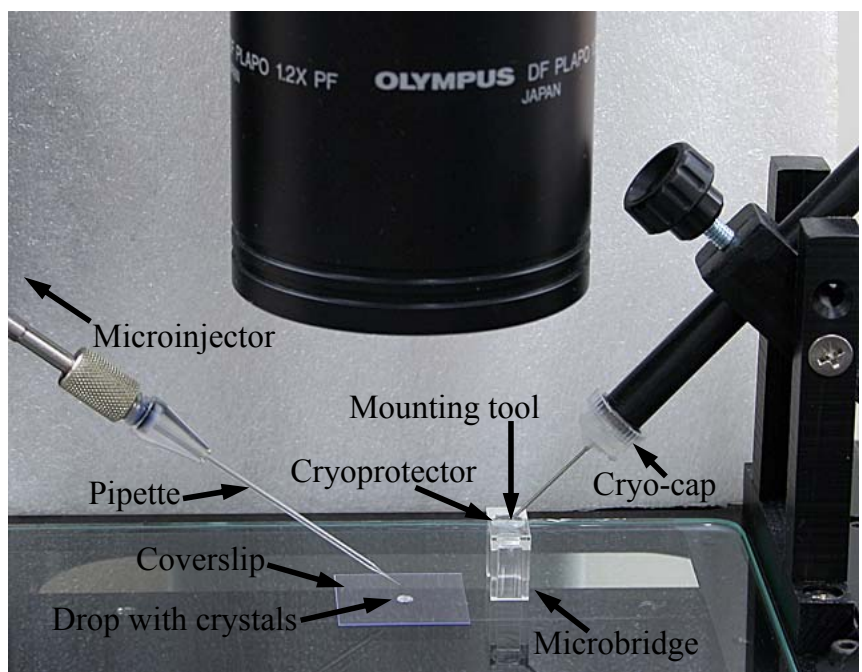

Fig. 4. A closeup of the workspace showing the pipette attached to the micromanipulator and the microinjector on the left side and the mounting tool attached to the receptor fixture on the right side of the microscope objective.

view of the microscope. The micromanipulator is a Sutter MP-285 - a 3-DOF Cartesian robot with a resolution of $40 \mathrm{~nm}$. A glass micropipette is mounted as the end-effector of the micromanipulator and is connected via a flexible plastic tube to the microinjector for control of pressure/suction. The microinjector we use is the oil-based CellTram Vario by Eppendorf which can exert pressure of up to $20,000 \mathrm{hPa}$ and has a minimum adjustment volume of $0.002 \mu$. This microinjector is designed for manual control via a rotating knob at its end; for computer control, we have had it retrofitted with a stepper motor in place of the knob.

We use a micromount as a crystal mounting tool, because of its numerous advantages described in the previous section, including its markings for ease of beamline mounting and alignment. The micromount comes attached to a metal pin, which we glue to a standard cryogenic cap the same way a loop is normally glued to it. From this point on, the micromount can be installed everywhere a loop is normally used (e.g. in a cryo-vial for flash freezing or on the goniometer of the synchrotron) since it is the cap that serves as the attachment mechanism. The cap has a small metal plate at the base which is how it is held on the goniometer's magnetic head.

For the task we target in this work, the micromount is held by a structure, we call receptor fixture (Fig. 3), which we have custom-designed and built for this specific purpose. The fixture allows for $4 \mathrm{DOF}$ of manual control for appropriate positioning of the tool. The tool itself is attached via the cap to the magnetic end of a plastic wand extending from the fixture (Fig. 4). The tool tip is adjusted for position and orientation so it is immersed in the cryoprotector and secured in place before the system starts. The receptor fixture is mounted directly onto the motorized stage, so that the micromount remains stationary with respect to the cryoprotector drop even when the stage moves. 


\section{SySTEM OPERATION}

The crystal mounting procedure starts with the placement of the necessary tools and objects in the workstation (Fig. 4): First, a microbridge with cryoprotector is placed at its designated location on the tray. Next a micromount is installed on the receptor fixture and is positioned adequately so it is immersed in the cryoprotector at an angle of approximately $45^{\circ}$ and is ready to receive the crystal. Finally, the user places a coverslip with the droplet containing the protein crystals on the microscope tray such that they are in the field of view.

The program is started next, which asks the user to specify which crystal among the possibly many in the drop is to be mounted. The user does this by selecting a rectangular region of interest (ROI) around the crystal on the screen and the automated process begins. The following steps are performed:

1) An ROI tracker is initialized with the ROI specified by the user in order to track the location of the crystal.

2) The micromanipulator is moved so that it lowers the tip of the pipette into the drop with crystals and positions it just to the left of the target crystal and at the appropriate height. A control loop with visual feedback is used to adjust the approach of the pipette, correcting for any motion of the crystal caused by the approach. The dip angle of the pipette tip is approximately $45^{\circ}$ with respect to the horizontal stage.

3) A control loop is established with visual feedback, such that it tracks the motion of the crystal and adjusts the suction of the microinjector correspondingly until the crystal is drawn inside the pipette. The loop completes when the crystal reaches a certain distance inside the pipette, which we refer to as the reference point.

4) The micromanipulator is moved up to withdraw the pipette from the drop and take it above the height of the microbridge.

5) The stage moves horizontally to position the microbridge in the field of view, adjusting the microscope focus accordingly. The pipette tip is now directly above the microbridge.

6) The pipette tip is immersed into the cryoprotector and positioned directly across the crystal aperture of the micromount.

7) The crystal is dispensed from the pipette onto the micromount by expelling all the liquid drawn from the drop in step 3. It is kept in the cryoprotector for as long as the crystallographer determines is necessary for best results. Care needs to be taken here to expel exactly the same amount of liquid that was taken in step 3 to avoid accumulation of the remainder in the pipette.

8) The micromount is withdrawn from the cryoprotector along with the crystal.

With the exception of steps 2 and 3 above, the rest of the steps are performed in open-loop fashion, because the system is calibrated for the locations and heights of the relevant objects and the system actuators meet the requirements for

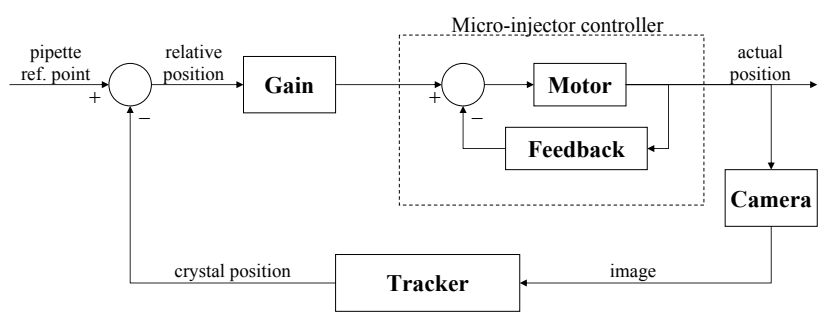

Fig. 5. Block diagram of the control system for crystal aspiration.

positioning accuracy. Steps 2 and 3 are closed-loop as they are using visual tracking to determine the location of the selected crystal and, based on that, to adjust the location of the pipette tip during approach (step 2) or the micronjector suction during aspiration (step 3). The control algorithms for steps 2 and 3 are completely analogous. The block diagram of the control system for crystal aspiration (step 3) is shown in Fig. 5; the difference for step 2 is that the reference point is just in front of the pipette tip (instead of inside it) and the controlled variable is the horizontal motion of the manipulator instead of the suction of the microinjector. The ROI tracking is based on sum of squared differences of pixel intensities and is efficiently performed by the XVision software package [27].

The main advantage of this method for crystal mounting consists of the separation of crystal pickup from cryoprotection and freezing. The pickup step is performed using a glass pipette, which is both more robust and less damaging to the crystal compared to when using traditional tools, such as cryogenic loops. It is more robust, because with the flow of liquid generated when suction is applied by the microinjector, it is easier to ensure that the crystal will follow into the pipette. Also, once inside the pipette, it will stably remain there. The phenomenon which is holding a crystal inside a loop or a micromount is surface tension, however it is stable only when the tool is taken out of the liquid; inside the drop the crystal can easily move about and capturing it requires excellent dexterity, precision and speed. It can be quite difficult at times for even adept crystallographers to perform the task manually. Duplicating their skills algorithmically would be enormously challenging. This issue is avoided by the use of a pipette for capturing the crystal.

Using a pipette is also less damaging to the crystal because the crystal never gets directly exposed to room conditions. The cryogenic loop, the microshovel and the micromount are open tools which expose the crystal to the surrounding environment. This comes as an advantage later in the pipeline, during the cryo-freezing step (Fig. 1, bottom center), when the crystal is exposed to low-temperatures (e.g. liquid propane), because an essential requirement for flash-freezing is the rapid drop of the crystal temperature. However, the open exposure of the crystal is a problem when it is transferred from the growth solution to the cryoprotector: any exposure to room conditions can be damaging to the crystal because of rapid dehydration. Even an exposure of 
only three seconds may prove to be too long and deteriorate its quality significantly. When the crystal is inside the pipette along with some of its reservoir solution, however, it is well protected from quick dehydration and remains in good condition until deposited onto the mounting tool.

On the other hand, while glass tubing can and has been used for both crystal mounting and X-ray imaging, as well as all stages in between, it poses its own unique problems. One is that very often excessive amount of liquid is drawn into the tube which later needs to be wicked out and this is a very challenging task. Excess liquid is usually not a big issue with loops and micromounts; micromounts even have a specially designated wicking hole. Another problem with glass tubes is that the crystal inside is very difficult to flashfreeze and this is for the same reason that they are good at preventing dehydration - insulation.

Given the above considerations, the reasoning behind our two-stage approach becomes obvious: we make use of the strengths of both glass tubes and micromounts while avoiding their weaknesses. We use pipettes for robustly capturing the crystal and safely transporting it across to the cryoprotector; next we transfer the crystal onto a micromount which is better suited to ensure good cryoprotection and speedy flash-freezing.

\section{EXPERIMENTS}

We have tested the system with different objects in environments with varying properties. For development and initial testing, we have used certified NIST traceable latex beads of nominal size $43 \mu \mathrm{m}$. Afterwards, tests have been performed using two types of protein crystals. One of these is the Haemophilus influenzae hypothetical protein HI1161 with reservoir solution consisting of 15-20\% PEG $3350(\mathrm{w} / \mathrm{v})$, $0.2 \mathrm{M}$ KFormate. The other is the enzyme Glucose Isomerase in $0.91 \mathrm{M}(\mathrm{NH} 4) 2 \mathrm{SO} 4,1 \mathrm{mM} \mathrm{MgSO} 4$. The cryoprotector used was $20 \%$ Ethylene Glycol.

A sample run of the system using Glucose Isomerase is shown in Fig. 6. The figure consists of six images illustrating six key steps of the process as viewed from the microscope. The first image is the initial configuration of the crystals in the drop. The crystal near the center is the one which is selected for mounting. Following is a snapshot taken after the pipette tip has been positioned to take the crystal (Fig. 6b). The next image, Fig. $6 c$, captures the moment when the microinjector is activated and is drawing the crystal inside the pipette. In Fig. 6d, the system has transitioned over to the micromount immersed in the cryoprotector and the pipette tip is positioned to dispense the crystal. Fig. 6e captures the moment when the crystal is being dispensed onto the micromount. Finally, Fig. 6f shows that the crystal has been successfully transferred onto the micromount.

Notice that the locations of some of the crystals (including the target) has changed. This is due to the disturbance caused by the pipette entering the drop. The selected crystal's location, however, is tracked by the camera and the pipette's approach is adjusted. This is another reason why visual tracking of the crystal is necessary.
The typical time it takes the system to execute a single run is 10-15 seconds. This compares favorably to the time crystallographers need to manually perform the task, which can range from a 2-3 seconds to minutes.

\section{CONCLUSION AND FUtURE WORK}

We have presented a functional robotic system for protein crystal mounting based on a micropositioner, a microinjector, a motorized stage and an optical microscope with a camera for visual feedback, and software control through a personal computer. We present a two-stage solution which combines the crystal mounting and cryoprotection steps and is designed to increase the robustness of the pickup as well as better preserving the crystal. The two-stage approach makes use of the strengths of both glass tubing and micromounts while avoiding their weaknesses. The glass pipette allows for robustly capturing the crystal and safely transporting it across to the cryoprotector while the micromount, where the crystal is eventually deposited, is better suited to ensure good cryoprotection and speedy flash-freezing.

As we have stated in the introduction, crystal mounting is only one in a series of steps taken to have the selected crystal cryogenically frozen and installed on a synchrotron for X-ray data collection. In an ideal scenario for a fully robotic high-throughput pipeline, the entire series of steps will be automated. The ultimate goal of our work is such automation, however, since automated solutions have already been proposed for the task of installing and aligning the crystal on a synchrotron (see Section II), in this work, we are mainly interested in transferring the crystal into the micromount while cryoprotecting it. In the next phase of our work, we will replace the receptor fixture with a manipulator which will be able to perform the cryogenic freezing and storage and allow the system to be integrated with existing beamline mounting technology.

\section{ACKNOWLEDGMENTS}

The authors wish to thank Prof. John Hunt and his group from the Biological Sciences Department at Columbia University for their help with crystallography as well as Matei Ciocarlie for his help with photography and suggestions. This work was supported by a grant to the Northeast Structural Genomics Consortium from the Protein Structure Initiative of the National Institute of Health (U54 GM074958).

\section{REFERENCES}

[1] F. Collins, M. Morgan, and A. Patrinos, "The human genome project: Lessons from large-scale biology," Science, vol. 300, no. 5617, pp. 286-290, April 2003.

[2] R. Service, "Structural genomics, round 2," Science, vol. 307, pp. 1554-1557, March 2005.

[3] C. L. Verlinde, "Structure-based drug design: progress, results and challenges," Structure 2, pp. 577-587, 1994.

[4] A. Georgiev, P. K. Allen, and W. Edstrom, "Visually-guided protein crystal manipulation using micromachined silicon tools," in Proc. IEEE/RSJ Int. Conf. on Intelligent Robots and Systems, IROS'04, September 2004, pp. 236-241.

[5] B.H.Weber and P.E.Goodkin, "A modified microdiffusion procedure for the growth of single protein crystals by concentration-gradient equilibrium dialysis," Archives of Biochemistry and Biophysics, vol. 141, pp. 489-498, 1970. 


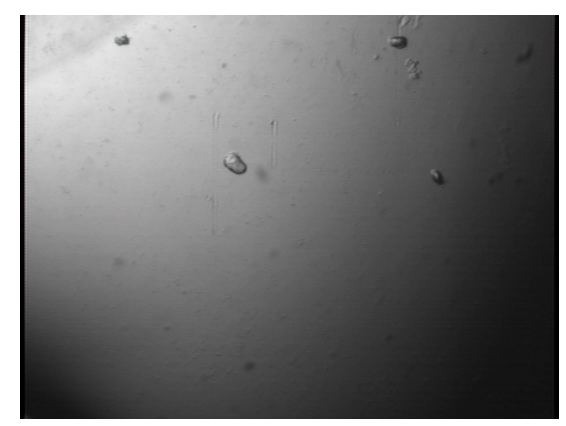

(a)

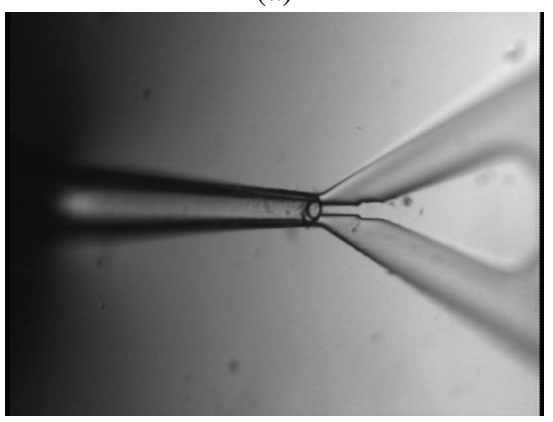

(d)

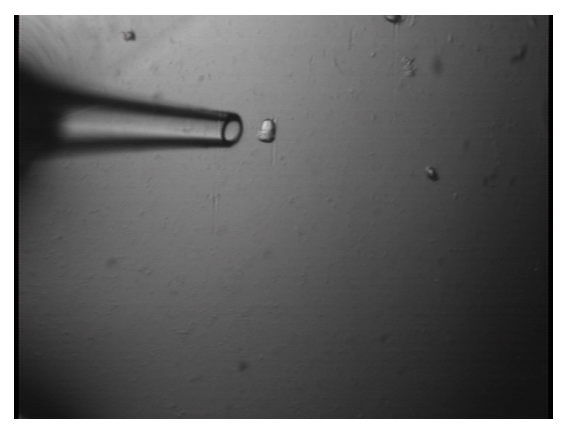

(b)

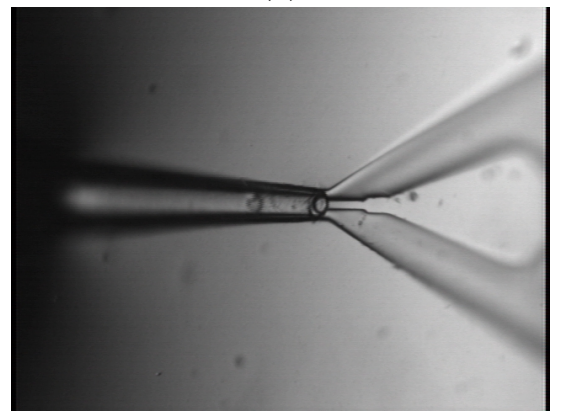

(e)

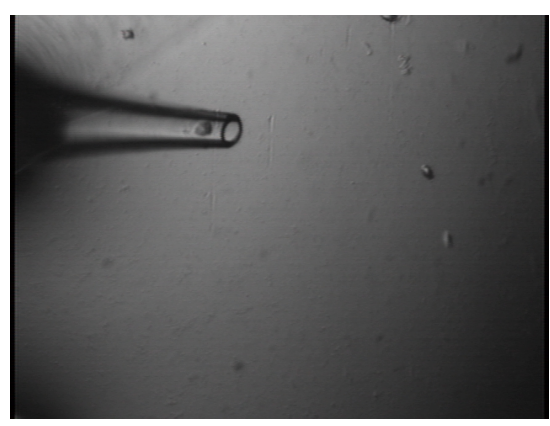

(c)

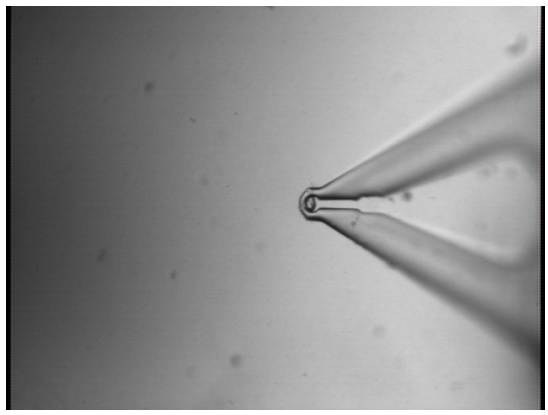

(f)

Fig. 6. Experimental test of the system: (a) initial view of the drop with crystals; (b) the pipette tip has descended into the drop and is positioned to capture the crystal to the right; (c) the crystal is drawn into the pipette; (d) the system transitions over to the micromount in the cryoprotector and the pipette tip is positioned next to the micromount; (e) the pipette is about to dispense the crystal; (f) the crystal has been successfully transferred onto the micromount and the pipette has withdrawn from the cryoprotector.

[6] F.R.Salemne, "A free interface diffusion technique for the crystallization of proteins for x-ray crystallography," Archives of Biochemistry and Biophysics, vol. 151, pp. 533-539, 1972.

[7] T.-Y.Teng, "Mounting of crystals for macromolecular crystallography in a free-standing thin film," Journal of Applied Crystallography, vol. 23, pp. 387-391, 1990.

[8] R. Thorne, Z. Sturm, J. Kmetko, K.O'Neil, and R. Gillilan, "Microfabricated mounts for high-throughput macromolecular cryocrystallography," Journal of Applied Crystallography, vol. 36, pp. 1455-1460, 2003.

[9] A. Georgiev, S. Vorobiev, W. Edstrom, T. Song, A. Laine, J. Hunt, and P. K. Allen, "Automated streak seeding with micromachined silicon tools," Acta Crystallographica Section D, vol. 62, pp. 1039-1045, 2006.

[10] D. Meldrum, M. Holl, C. Fisher, M. Saini, S. McGuire, T. Ren, W. Pence, S. Moody, D. Cunningham, D. Donaldson, and P. Wiktor, "Sample preparation in glass capillaries for high-throughput biochemical analyses," in Proc. Int. Conf. on Automation Science and Engineering, CASE'05, 2005, pp. 7-12.

[11] G. Snell, C. Cork, R. Nordmeyer, E. Cornell, G. Meigs, D. Yegian, J. Jaklevic, J. Jin, R. Stevens, and T. Earnest, "Automated sample mounting and alignment system for biological crystallography at a synchrotron source," Structure, vol. 12, pp. 537-545, April 2004.

[12] Y. Kalinin, J. Kmetko, A. Bartnik, A. Stewart, R. Gillilan, E. Lobkovsky, and R. Thorne, "A new sample mounting technique for room-temperature macromolecular crystallography," Journal of Applied Crystallography, vol. 38, pp. 333-339, 2005.

[13] B. Hannaford, J. Hewitt, T. Maneewarn, S. Venema, M. Appleby, and R. Ehresman, "Telerobotic remote handling of protein crystals," in Proc. IEEE Int. Conf. on Robotics and Automation, ICRA'97, 1997, pp. 1010-1015.

[14] H.-Y. Chan and W. J. Li, "A thermally actuated polymer micro robotic gripper for manipulation of biological cells," in Proc. IEEE International Conference on Robotics and Automation, September 2003, pp. 288-293.

[15] F. Arai, T. Sakami, H. Maruyama, A. Ichikawa, and T. Fukuda, "Minimally invasive micromanipulation of microbe by laser trapped micro tools," in Proc. IEEE International Conference on Robotics and Automation, Washington, DC, May 2002, pp. 1937-1942.
[16] S. Haliyo, S. Regnier, and J.-C. Guinot, "[mü]mad, the adhesion based dynamic micro-manipulator," European Journal of Mechanics A/Solids, vol. 22, no. 6, pp. 903-916, November 2003.

[17] S. Hutchinson, G. Hager, and P. Corke, "A tutorial on visual servo control," IEEE Trans. on Robotics and Automation, vol. 12, no. 5, pp. 651-670, October 1996.

[18] P. K. Allen, A. Timcenko, Y. B., and P. Michelman, "Automated tracking and grasping of a moving object with a robotic hand-eye system," IEEE Trans. on Robotics and Automation, vol. 9, no. 2, pp. 152-165, April 1993.

[19] J. T. Feddema and T. R. Christenson, "Parallel assembly of high aspect ratio microstructures," in SPIE Conference on Microrobotics and Micromanipulation, Boston, USA, September 1999, pp. 153-164.

[20] B. J. Nelson, Y. Zhou, and B. Vikramaditya, "Sensor-based microassembly of hybrid mems devices," IEEE J. of Control System, pp. 35-45, December 1998.

[21] S. Yu and B. J. Nelson, "Autonomous injection of biological cells using visual servoing," The International Journal of Robotics Research (IJRR), vol. 21, no. 10-11, pp. 861-868, October-November 2002.

[22] K. Hashimoto, Visual Servoing : Real Time Control of Robot Manipulators Based on Visual Sensory Feedback. World Scientific Series in Robotics and Automated Systems, Vol 7, World Scientific Press, Singapor, 1993.

[23] L. Weiss, A. Sanderson, and C. Neuman, "Dynamic sensor-based control of robots with visual feedback," IEEE Journal of Robotics and Automation, vol. 3, no. 5, pp. 404-417, October 1987.

[24] P. Martinet, N. Daucher, J. Gallice, and M. Dhome, Robot control using monocular pose estimation. Worshop on New Trends in Imagebased Robot Servoing, IROS'97, Grenoble, 1997.

[25] W. Wilson, C. W. Hulls, and G. Bell, "Relative end-effector control using cartesian position-based visual servoing," IEEE Trans. on Robotics and Automation, vol. 12, no. 5, pp. 684-696, 1996.

[26] F. Chaumette, "Potential problems of stability and convergence in image-based and position-based visual servoing," The Confluence of Vision and Control, D. Kriegman, G. Hager, A. Morse (eds), LNCIS Series, Springer Verlag, vol. 237, pp. 66-78, 1998.

[27] G. Hager and K. Toyama, "The xvision system: A portable substrate for real-time vision applications," Computer Vision and Image Understanding, vol. 69, no. 1, pp. 23 - 37, 1998. 